\title{
Static, Dynamic and Stereological Testing of 3D-Printed Acetabular Cups: A Mechanical Assessment of Acetabular Cup Porous Structures
}

\section{ISSN: 2576-8875}

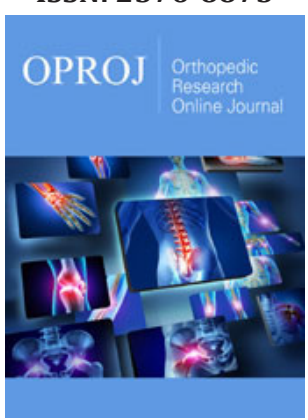

*Corresponding author: Kyle A McGrath, Ohio University Heritage College of Osteopathic Medicine, Dublin, USA

Submission: 侮 September 14, 2021

Published: 傮September 27, 2021

Volume 8 - Issue 5

How to cite this article: Kyle A McGrath*, Hayley Flynn, Mark Gittins, Garrett Spurgeon, Cowan Moore. Static, Dynamic and Stereological Testing of 3D-Printed Acetabular Cups: A Mechanical Assessment of Acetabular Cup Porous Structures. Ortho Res Online J. 8(5). OPROJ. 000699. 2021.

DOI: $10.31031 /$ OPROJ.2021.08.000699

Copyright@: Kyle A McGrath, This article is distributed under the terms of the Creative Commons Attribution 4.0 International License, which permits unrestricted use and redistribution provided that the original author and source are credited.

\author{
Kyle A McGrath ${ }^{1 *}$, Hayley Flynn ${ }^{1}$, Mark Gittins ${ }^{2}$, Garrett Spurgeon ${ }^{1}$ and Cowan \\ Moore $^{1}$ \\ ${ }^{1}$ Ohio University Heritage College of Osteopathic Medicine, Dublin, USA
}

${ }^{2}$ Associate Clinical Professor of Orthopedic Surgery, Ohio University Heritage College of Osteopathic Medicine, Dublin, USA

\begin{abstract}
Introduction: Cementless Total Hip Arthroplasty (THA) is a surgical procedure commonly used in the treatment of osteoarthritis of the hip. Implant manufacturers typically apply porous coatings (e.g. beads or plasma-sprayed titanium) to acetabular cup substrates to promote bone ingrowth but have recently begun to leverage additive manufacturing (AM) or "3D printing." AM enables implant designers to create porous structures that were previously not possible, as the structures are "grown" from the substrate, not applied. The goal of this study is to compare design features and mechanical properties of AM and non-AM acetabular cups.
\end{abstract}

Methods: Two-point fatigue, static shear, static tensile, shear fatigue, plastic deformation, and particle debris tests were performed on AM and non-AM acetabular cups.

Results: Two-point fatigue endurance limits for AM and non-AM cups were $1300 \mathrm{~N}$ and $1100 \mathrm{~N}$, respectively $(\mathrm{p}<0.001)$. There was no measurable difference in weight or debris loss for the AM and non-AM acetabular cups after 2.5 million cycles of two-point fatigue loading at $1500 \mathrm{~N}$. The AM porous structure exceeded acceptance criteria in all testing - shear fatigue strength, static shear strength, static tensile strength, and plastic deformation.

Conclusion: The fatigue strength, minimal debris shedding, and stereological properties of the AM acetabular cup, along with design features exclusive to this manufacturing method may suggest that additive manufacturing can produce implants that are equivalent or superior to traditional methods. Further research is needed to assess whether additive manufacturing can lead to favorable implant stability and patient outcomes.

Keywords: Total hip arthroplasty; Acetabular component; Additive manufacturing

\section{Introduction}

Cementless Total Hip Arthroplasty (THA) is a surgical procedure commonly used in the treatmentof osteoarthritis of the hip. Conventional prosthesis used in THA involves a femoral stem, a spherical metallic or porcelain cap to serve as the femoral head and an acetabular component which consists of a metallic acetabular cup and an inner polyethelene liner in which the femoral head component fits. The acetabular cup (Figure 1) has traditionally been produced through a process known as subtractive manufacturing wherein implants are machined from forged blanksor bar stock and coated in either plasma sprayed titanium (TPS) or sintered beads. Recent trendsin metallic orthopedic implant manufacturing, however, have begun a shift towards additive manufacturing (AM) or "3D printing" as a means of production away from the traditional production method. Additive manufacturing allows for a more sophisticated level of implant design with the goal of improving in-vivo stability without sacrificing structural integrity [1]. Various features of additive manufacturing are described in Figure 1; [1]. Additive manufacturing has opened numerous doors of opportunity in the orthopedic world. From implants and prostheses to surgical instruments and anatomic modeling, additive manufacturing has revolutionized the customizability of orthopedic surgery today and redefined its future direction $[2,3]$. 


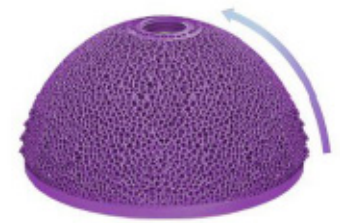

A. Gradient Porosity

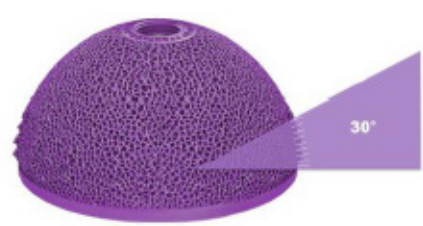

B. Perpendicular Raised Struts (first $30^{\circ}$ )
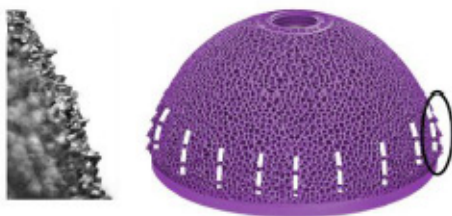

C. Anti-Rotational Fins

\section{Figure 1:}

The process of additive manufacturing typically begins with a Computer Aided Design (CAD) ofa desired three-dimensional (3D) component, which is then given to a machine capable of laying down layers of polymer-based powder which can then be converted into the metallic end product through a series of thermochemical reactions. This process allows for micron-level customization, and for components to be produced from a "bottom-up" approach rather than a "top-down" approach seen when machining components from solid metal [1].

While additive manufacturing has gained traction over the last several years, the literature surrounding additive manufactured acetabular cups is sparce, with no studies to date assessing the mechanical integrity of AM acetabular cups to traditionally manufactured cups on a mechanical level. Because these components are used as orthopedic prosthetic implants, the mechanical integrity of acetabular cups can have significant implications on clinical outcome in those receiving THA. Therefore, given the growing popularity of additive manufacturing, this knowledge gap should be addressed. Herein we conducted a series of tests comparing the mechanical and stereological properties of AM acetabular cups to those of traditionally manufactured cups commonly being used today.

\section{Methods}

This study was made possible by the Orthopedic Foundation Summer Research Grant, Next Step Arthropedix, and Ohio University Heritage College of Osteopathic Medicine. This was a mechanical study on metallic acetabular cup components taking place from January 2018 to October 2018. Institutional review board and ethics committee approval were not required giventhe nature of this study.

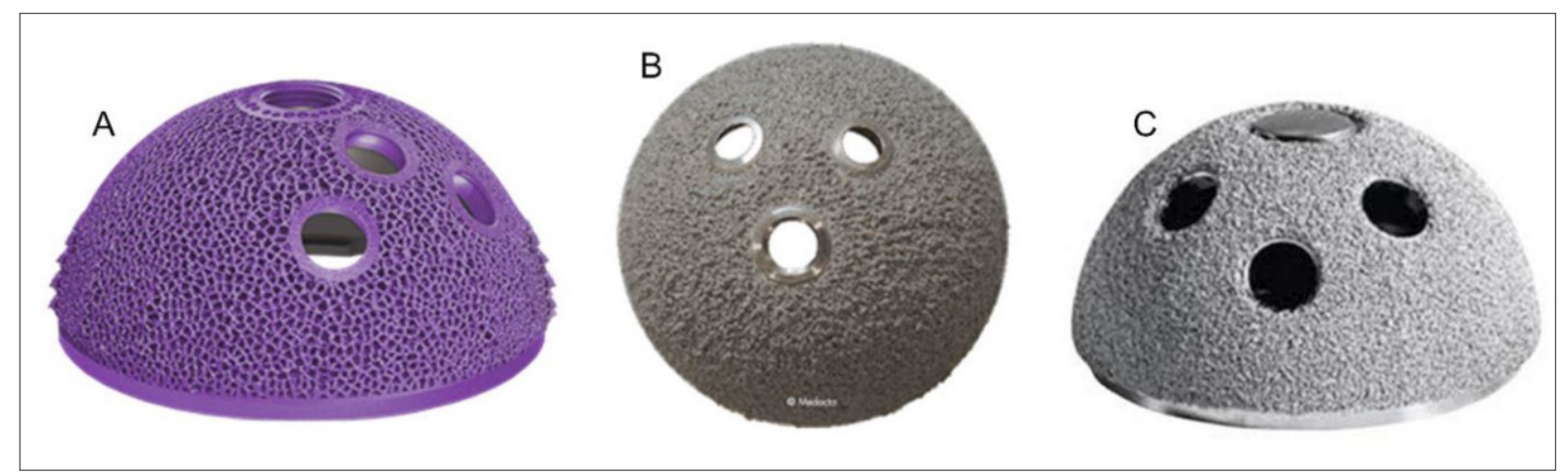

Figure 2:

The AM implant components were evaluated with a series of static, dynamic, and stereological methods. The AM cup's porous structure was specifically tested in static shear, static tensile, shear fatigue, and plastic deformation in accordance with American Society of Testing and Materials (ASTM) consensus standards. ASTM employs these standard for implants containing porous structures to ensure that these implants are mechanically sound, as porous metallic implants are structurally and mechanically very different from and presumably weaker than solidmetallic implants for instance. Two-point fatigue and particle debris shedding properties were also assessed using established methods ${ }^{2}$ to compare cups manufactured by AM and traditional methods. Stereological evaluations of the AM cups were also performed and compared to ASTM consensus standards. The subject cups were selected based on differences in their manufacturing process (e.g. AM vs. subtractive manufactured cups with applied coatings). The AM acetabular Cups (Figure 2A) are produced via electron beam melting additive manufacturing (Arcam, GE), wherein acetabular 
components are grown from consecutive layers of Ti-6Al-4V ELI powder (50micron, AP\&C, Boisbriand, QC, Canada). This process allows the manufacturer to design the component to desired specifications with precision equal to that of the size of the titanium substrate particles. The AM cup possesses a porous surface design with anti-rotational features that is intended to mimic the native cortical and cancellous bone of the reamed acetabulum. In contrast, traditionally manufactured cups feature a plasma sprayed or sintered bead coating, providing a coarse textured surface without any additional geometrical features that may aid in stability. Two types of traditionally manufactured cups were used. The first uses a forged titanium alloy cup substrate with an applied titanium plasma spray coating (Figure 2B). The second cup uses a forged titanium alloy cup with a sintered bead coating (Figure 2C). The AM cup was compared to each of the traditionally manufactured cup designs with applied coatings.
All specimens were of finished implant quality and remained in original packaging untilimmediately prior to testing.

\section{Testing}

Fatigue and debris shed testing: The testing apparatus used was a single axis load frame (meeting requirements of ASTM E 467) capable of generating constant compression, traction, and sinusoidal oscillating force to an accuracy of $\pm 2.0 \%$ of the peak compressive force, without restraint forces in the horizontal plane (meeting requirements for ISO 7206-4). Debris shed testing consisted of testing three samples (one AM cup, one plasma sprayed cup, and one sintered bead coated cup), while fatigue testing consisted of 17 cups (nine AM cups and eight traditionally manufactured cups). For fatigue and debris shed testing the loading apparatus was designed to maintain a two-point contact with the outer rim of the cup when loaded (Figure 3D); [4].
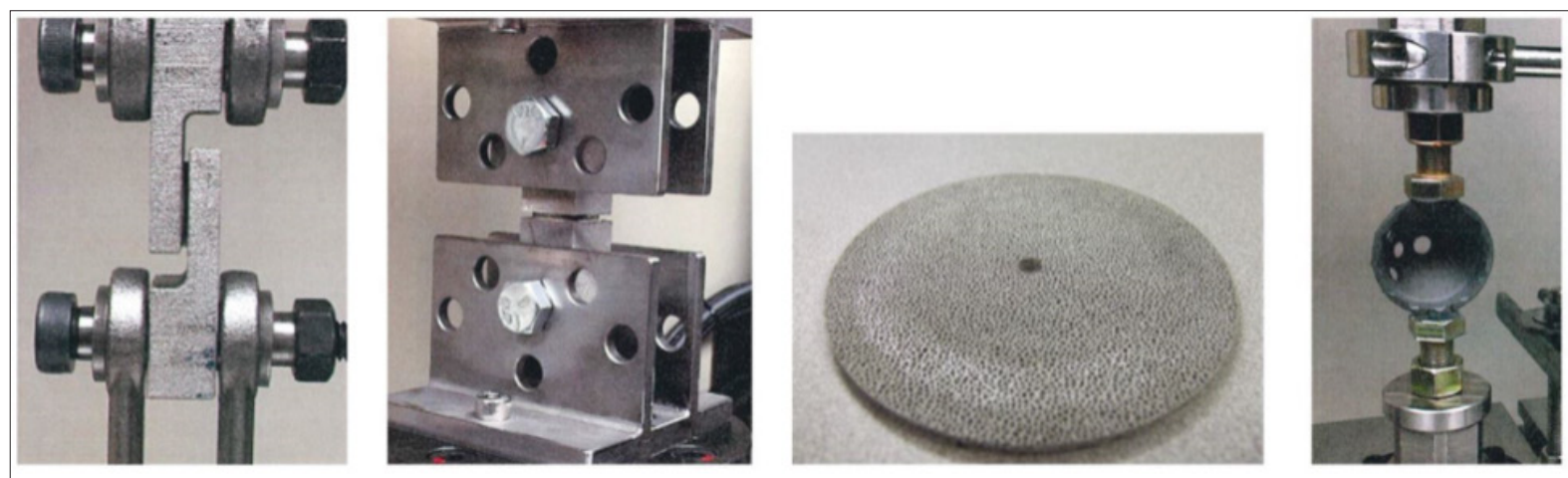

Figure 3:

Fatigue loading and particle debris testing was performed in serial, beginning with the removal of the cup to be tested from its native packaging. A dry mass was obtained followed by consecutive rounds of cleaning until a stable mass within a range of $0.0005 \mathrm{~g}$ between consecutive weighing was achieved. The test cup was then placed in the pre-calibrated test machine (MTS 810 Axial-Torsion Bionix Frame) to undergo static and dynamic axial compressions. Fatigue loading consisted of increasing compressive force on the cup until the maximum force $(\mathrm{N})$ was reached at which the cup could withstand virtually infinite compressive cycles $(5$ million cycles at $15 \mathrm{~Hz}$ ) without failing. Due to the nature of fatigue testing, (determining yield strength) each cup could only be tested once, indicating that 17 rounds of testing were done $(n=17)$. Debris shed testing consisted of the same compressive apparatus described for the fatigue testing, however $1500 \mathrm{~N}$ for 2,500,000 cycles at $15 \mathrm{~Hz}$ was used (an active person on average takes an estimated 2 million steps per year [5]). Dry mass analysis of the cup, visual cup inspection, and debris collection and analysis were all performed at intervals of $0.25,0.5,1.25$, and 2.5 million cycles. This method of compression testing was designed to expose acetabular cups to similar compressive forces that they may experience under repeated stress in vivo. Dry mass analysis was preceded with cleaning via a validated cleaning protocol to remove loose debris. Cups were weighed and immediately returned to the test apparatus or stored in a dry area to prevent possible aerosol particulate collection and weight gain. Cup inspection was performed, and visible signs of debris loss, fractures, or other signs of fatigue were recorded.

Porous structure testing: Test samples utilized for porous structure testing consisted of a single additively manufactured testing component, connected on either end by the force-loading frame and joined at the focal point by the same trabecular framework employed on the articulating surface of the acetabular cups. This ensured that the structural integrity of the trabecular framework could be isolated andtested. For all porous structure testing (static shear, static tensile, shear fatigue, and plastic deformation) (Figure 3 ), compressive and/or tensile forces were applied to determine the maximum stresses or elongations that could be endured before failure. For this testing protocol, additive manufacturing was utilized in producing the testing components. For shear and tensile testing, the authors 3D printed a single component with mating joints on either end to fit the testing apparatus. The middle of the testing component was composed only of the porous trabecular structure used on the outer surface of the acetabular cup. Because AM was used, the component could be produced as one contiguous piece, and the shear force could be isolated to the trabecular structure as described, allowing for a more accurate assessment of its integrity. For abrasion testing, an AM disc with a surface consisting of a 
trabecular structure underwent continuous abrasion with an axially rotating titanium component oriented perpendicular to the disc, in which the normal force (mg) at which the trabecular structure began to deform was determined as the threshold for abrasion. The data gathered was compared to benchmarks outlined in ASTM standards for evaluating porous coatings on medical implants.

\section{Stereological evaluation}

Stereological evaluation, done on one pre-packaged AM cup, included diameter/width of the pores, minimum void intercept length or minimum pore diameter, and mean volume percent of voids

\section{Statistical analysis}

All computations were done in $\mathrm{R}$, version 3.5.0 (R Foundation, Vienna, Austria) and p-values less than 0.05 were considered statistically significant. Numerical variables were compared using Independent T tests. Due to the exploratory nature of this study, we did not correct for multiple testing but interpreted our findings in light of the several measures being compared.

\section{Results}

\section{Porous structure testing}

One testing component was used in each type of mechanical analysis. The results of AM porous structure testing are listed in Table 1 . The additively manufactured porous structure exceeded ASTM acceptance criteria (AC) in all testing: shear fatigue strength ( $\geq 12 \mathrm{MPa}$; $\mathrm{AC} \geq 10 \mathrm{MPa}$ ), static shear strength (26.9MPa; $\mathrm{AC}=20 \mathrm{MPa}$ ), static tensile strength $(27.4 \mathrm{MPa} ; \mathrm{AC}=20 \mathrm{MPa})$, abrasion resistance (9.7 $\pm 2.2 \mathrm{mg} ; \mathrm{AC}<65 \mathrm{mg}$ ), and plastic deformation (77.2MPa) which has no established acceptance criteria by the ASTM.

Table 1: Porous structure testing of acetabular cups vs. ASTM Standards.

\begin{tabular}{|c|c|c|c|}
\hline Test & Method & Result & Acceptance Criteria \\
\hline Shear Fatigue Strength & ASTM F1160 & $\geq 12 \mathrm{MPa}$ & $20 \mathrm{MPa}$ \\
\hline Static Shear Strength & ASTM F1044 & $26.9 \mathrm{MPa}$ & $20 \mathrm{MPa}$ \\
\hline Static Tensile Strength & ASTM F1147 & $27.4 \mathrm{MPa}$ & $<65 \mathrm{mg}$ \\
\hline Abrasion Resistance & ASTM F1978-12 & $9.7 \pm 2.2 \mathrm{mg}$ & $77.2 \mathrm{MPa}$ \\
\hline Plastic Deformation & ASTM E9 & N/A \\
\hline
\end{tabular}

ASTM: American Society of Testing and Materials.

\section{Fatigue testing}

Endurance limits for fatigue load testing ( 5 million cycles at maximum force) was found to be1300N for the AM cup and $1100 \mathrm{~N}$ for the TPS cup ( $n=17, \mathrm{p}<0.001)$ (Table 2 \& Figure 4).

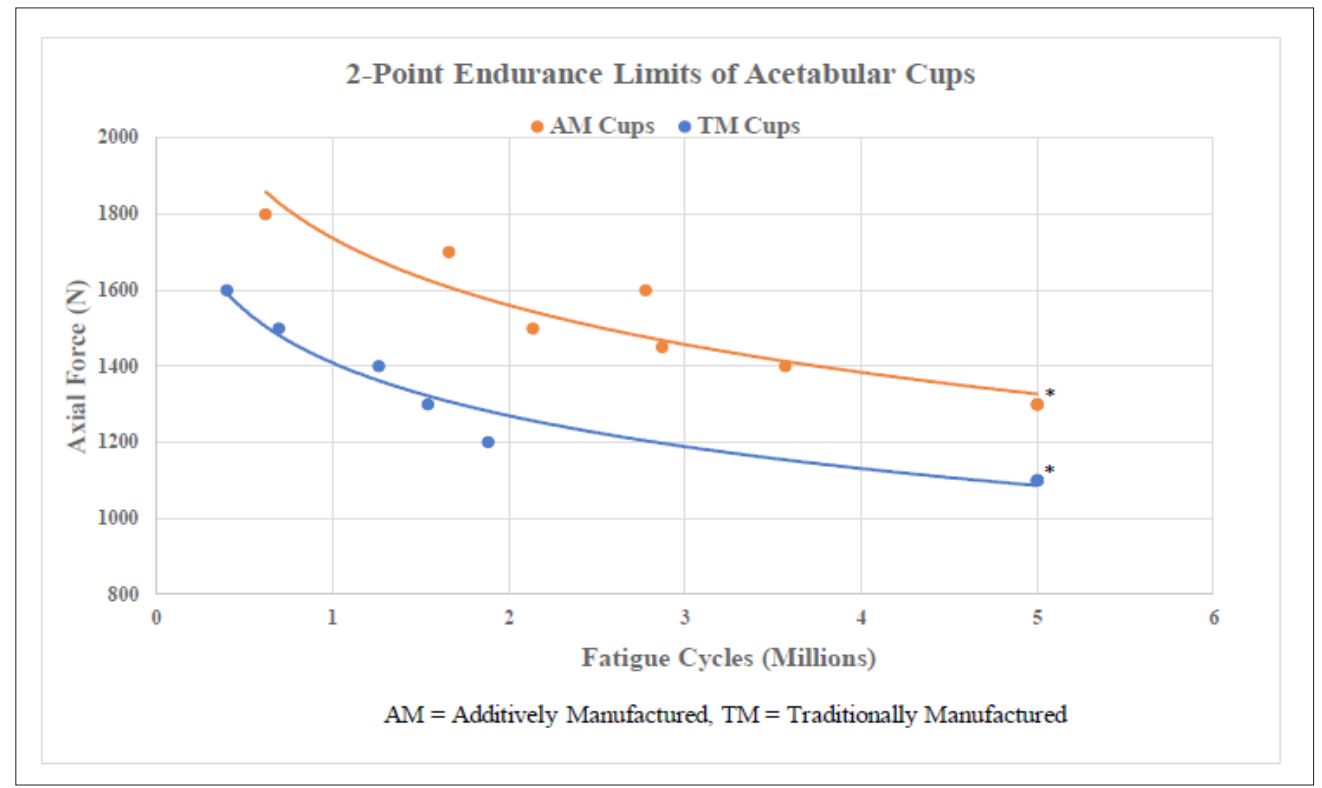

Figure 4: Two-point endurance limits of acetabular cups.

${ }^{*} \mathrm{p}<0.001$ 
Table 2: Two-point endurance limits of acetabular cups.

\begin{tabular}{|c|c|c|}
\hline Cup Model & Load (N) & 621062 \\
\hline NS-54-BL & 1800 & 1660333 \\
\hline NS-54-BL & 1700 & 2776447 \\
\hline NS-54-BL & 1600 & 2138575 \\
\hline NS-54-BL & 1500 & 3571210 \\
\hline NS-54-BL & 1450 & 50000000 \\
\hline NS-54-BL & 1400 & 5000000 \\
\hline NS-54-BL & 1300 & 5000000 \\
\hline NS-54-BL & 1300 & 400713 \\
\hline NS-54-BL & $1300^{*}$ & 694860 \\
\hline & & 1263421 \\
\hline CO-54-M & 1600 & 1543239 \\
\hline CO-54-M & 1500 & 1882704 \\
\hline CO-54-M & 1400 & 5000000 \\
\hline CO-54-M & 1300 & 5000000 \\
\hline CO-54-M & 1200 & 5000000 \\
\hline CO-54-M & 1100 & 1100 \\
\hline CO-54-M & $1100^{*}$ & \\
\hline CO-54-M & & \\
\hline
\end{tabular}

NS-54-BL: Additive manufactured cup model

CO-54-M: Traditionally manufactured cup model

${ }^{*} \mathrm{p}<0.001$

\section{Debris shedding}

Three identical AM cups were fatigue tested and compared to commercially available acetabularcups (Figure 3D). All acetabular

\section{Stereological evaluation - AM Cups}

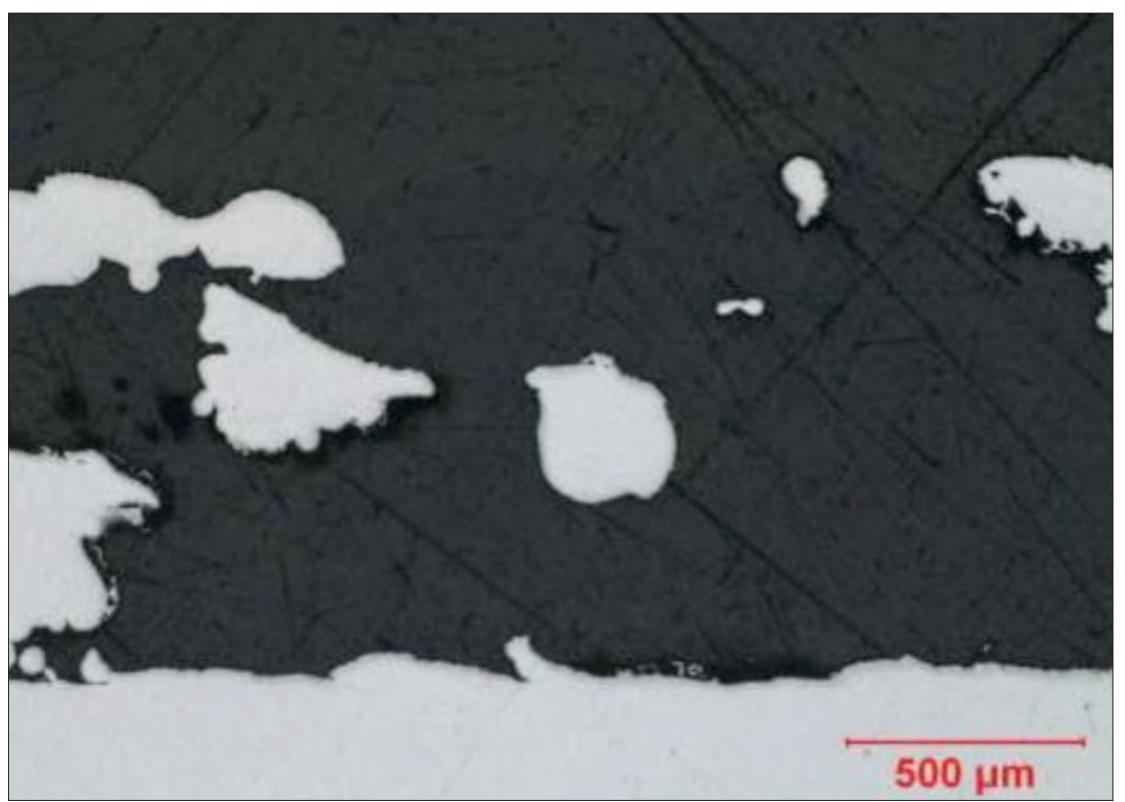

Figure 5:

components were weighed on analytical scales before and after fatigue testing. There was no measurable difference in weight for the TPS, beaded, or AM cup after 2.5 million cycles of two-point fatigue loading at $1500 \mathrm{~N}$. 
The porous structure of the AM cup has a variable gradient porosity ranging from least porous atthe rim to most porous at the apical dome (Figure 1). The porous structure was characterized in accordance with ASTM F1854-15, "Standard Test Method for Stereological Evaluation of Porous Coatings on Medical Implants" and found to be within specification for pore diameter, minimum void intercept length, and mean volume percent of voids (Figure 5); [6].

\section{Discussion}

As additive manufacturing is becoming more widely used in implant manufacturing, it is important to assess the integrity of these components, comparing them not only to components that have been in use for many years but to contemporary consensus standards. Mechanical failure and metallic debris shedding from THA implants has been studied significantly in the development and manufacturing of safer components. A study of almost 300,000 THA patients showed 10-year revision rates for cementless THA ranging from $3-5 \%$, and patients whose implants undergo even minimal metallic debris shedding following surgery may experience a variety of complications leading to revision surgery [7]. The extent to which loose metallic debris interacts with the surrounding tissues depends on the amount of delaminated porous structure and the morphology of the released metallic particles. Severe cases include inflammatory responses that cause tissue necrosis or pseudotumor formation, however the most commonly seen complication associated with metallic debridement in the joint is aseptic loosening of the prosthesis due osteolysis of the surrounding bone [8]. This is thought to occur primarily through increased macrophage expression of TNF- $\alpha$, a potent activator of osteoclastic activity and bone resorption [9]. While the parameters of this study are not directly concerned with the clinical implications of metallic debris shed, understanding the importance of mechanical integrity among metallic implants and the issues that can arise when implants break down in vivo is important when discussing the purpose of mechanical studies like this one. In mechanical testing of these acetabular cups, taking serial masses via analytical scales throughout testing was thoughtto be the best method of debris shed measurement in order to assure microscopic debris would beaccounted for. Identifying microscopic metallic debris shed was essential, as it has been demonstrated that nanoparticles from joint prostheses (able to be phagocytosed by joint macrophages) can directly disrupt cellular processes, increasing cytokine production and facilitating aseptic loosening of the implant [10].

In a series of many tests on both traditional and additively manufactured cups, we found that the AM cups demonstrated a higher fatigue run-out limit, as they were able to withstand $1300 \mathrm{~N}$ at 5million cycles without any noticeable failure or plastic deformation, while the traditionally manufactured cups tested were only able to withstand $1100 \mathrm{~N}$. Despite the small sample size of consumergrade cups beign tested $(\mathrm{n}=17)$, this difference in load tolerability was found to be statistically significant, suggesting that additive manufacturing, while a relatively new method ofcup manufacturing, may produce cups with equivalent or superior mechanical integrity to those produced via traditional manufacturing methods. Our testing also showed no measurable difference in mass lost (debris shed) among any of the cups when subject to $1500 \mathrm{~N}$ at $15 \mathrm{~Hz}$ out to 2.5 million cycles, suggesting that AM cups behave equivalent to traditional cups under similar cyclical stress. Furthermore, The AM cup's porous structure met or exceeded all benchmarks required by ASTM standards for stereological and mechanical properties. These results suggest that while additive manufacturing is a relatively novel method of acetabular cup production, it produces structurally sound components that can pave the way for futureinnovation and improvements in patient outcomes.

Looking ahead, the incorporation of patient imaging such as X-Ray, Computed Tomography (CT) and Magnetic Resonance (MR) may serve a valuable purpose as these have been utilized in the past to assist pre-operative 3D component design [11-13]. Regarding acetabular cups, additional image planning may be useful with planning more challenging THA cases in the setting of trauma or patients with complex acetabular architecture. Ultimately, the authors hope this study adds valuable insight into the growing body of literature surrounding the use of AM in orthopedic surgery.

\section{Limitations}

This study has limitations that must be addressed. First, our specimen sample size was relatively limited despite achieving statistical significance in fatigue testing. However, all implants tested were of manufactured standards and purchased as such, implying that each specimen should be similar on a mechanical and molecular level within a very small margin of error as the manufacturer intended. Because of the presumed consistency between specimens meeting final production standards, only one AM cup was evaluated against ASTM standards. Additionally, this was a mechanical study on innovative implant technology, meaning that our results cannot be extrapolated to clinical conclusions. However, while the data we present herein is not clinicalin nature, we believe it has strong implications in clinical research, and we hope this study is a catalyst for future outcome studies in patients with AM prostheses.

\section{Conclusion}

Additive manufacturing is a growing field that has recently become more widely employed in themanufacturing of orthopedic implant components. It is a relatively novel method of producing orthopedic implants, and it may provide substantial benefits in production efficiency and cost reduction. The present study demonstrates that additive manufacturing is a viable production method for metallic acetabular cups, producing implants that have similar or superior mechanical integrity than traditional manufactured cups. We believe there is great promise in the future of additive manufacturing and hope that our study prompts further research in this field and how it can benefit patient care. 


\section{References}

1. Javaid Mohd, Haleem A (2018) Additive manufacturing applications in orthopaedics: A review. Journalof Clinical Orthopaedics and Trauma 9(3): 202-206.

2. Wong KC (2016) 3D-printed patient-specific applications in orthopedics. Orthopedic Research and Reviews 8: 57-66.

3. Wong TY, Cheung CMG, Lai TYY (2019) Efficacy and safety of intravitreal aflibercept and ranibizumab in asian patients with neovascular agerelated macular degeneration. Retina. 39(3): 537-547.

4. Squire M, Griffin WL, Mason JB, Peindl RD, et al. (2006) Acetabular component deformation with press-fit fixation. The Journal of Arthroplasty 21(2): 306.

5. Silva M, Shepherd EF, Jackson WO, Dorey FJ, Schmalzried TP (2002) Average patient walking activity approaches 2 million cycles per year. The Journal of Arthroplasty 17(6): 693-697.

6. https://www.astm.org/Standards/F1854.htm

7. Kandala NB, Connock M, Pulikottil-Jacob R (2015) Setting benchmark revision rates for totalhip replacement: analysis of registry evidence. BMJ 350 (mar09 1): h756-h756.
8. Davis DL, Morrison JJ (2016) Hip arthroplasty pseudotumors: pathogenesis, imaging, and clinical decision making. Journal of Clinical Imaging Science 6: 17.

9. Nich C, Goodman SB (2014) Role of macrophages in the biological reaction to wear debris fromjoint replacements. Journal of Long-Term Effects of Medical Implants 24(4): 259-265.

10. Yao JJ, Lewallen EA, Trousdale WH (2017) Local cellular responses to titanium dioxide fromorthopedic implants. Bio Research Open Access 6(1): 94-103.

11. Javaid Mohd, Haleem A (2018) Additive manufacturing applications in medical cases: A literaturebased review. Alexandria Journal of Medicine 54(4): 411-422.

12. Srivastava A, Aggarwal AN, Mishra P, Bhateja D (2016) Femoral fracture acting as an "ominous masquerade" in a 7-year-old child. Journal of Clinical Orthopaedics and Trauma 7: 27-29.

13. Vaishya R, Vijay V, Krishnan M, Agarwal AK (2018) Fallacies of CT based component size prediction in total knee arthroplasty - Are patient specific instruments the answer? Journal of Clinical Orthopaedics and Trauma 9(1): 34-39. 